\title{
RELACIÓN ENTRE LA EXPOSICIÓN INFORMAL A UNA LENGUA EXTRANJERA (INGLÉS Y FRANCÉS) Y LA COMPETENCIA LÉXICA
}

\author{
INMACULADA CLOTILDE SANTOS DÍAZ \\ Universidad de Málaga, España \\ ESTER TRIGO IBÁÑNZZ \\ Universidad de Cádiz, España
}

MANUEL FRANCISCO ROMERO OLIVA

Universidad de Cádiz, España

\begin{abstract}
Resumen. El aprendizaje de una lengua extranjera se limita en ocasiones a un contexto académico. Sin embargo, la exposición a una lengua en un contexto informal, entendido como aquel que se realiza en un determinado medio social o en un país extranjero (Pendanx, 1998), juega un papel crucial tanto en la capacidad para aprender o adquirir la lengua como en la motivación intrínseca y extrínseca de los hablantes. El objetivo de este trabajo es mostrar la relación entre el léxico y el uso de una lengua extranjera (inglés o francés) en un contexto familiar. Los informantes, 171 estudiantes de postgrado de la Universidad de Málaga, contestaron a un cuestionario que recogía el número de horas semanales que usaban la lengua extranjera para comunicarse con familiares y amigos. Además, para evaluar el vocabulario, realizaron una prueba de disponibilidad léxica siguiendo las recomendaciones del Proyecto Panhispánico de Disponibilidad Léxica, en la que evocaron durante dos minutos palabras relacionadas con un tema o centro de interés. Los resultados muestran que se produce una correlación significativa positiva entre el vocabulario y las variables relacionadas con la exposición informal a la lengua extranjera. El coeficiente de correlación de Pearson entre el léxico y el uso de la lengua con amigos es de 0,323 y entre el léxico y el uso de la lengua con familiares es de 0,218 , en ambos casos a un nivel de significancia de 0,01 . Por ello, se corrobora la importancia del aprendizaje informal en el aprendizaje de una lengua extranjera (Krashen, 1987).
\end{abstract}

Palabras clave: aprendizaje de una lengua extranjera, contexto de aprendizaje, disponibilidad léxica, vocabulario 


\section{INTRODUCCIÓN}

En el proceso de adquisición y aprendizaje de una lengua intervienen numerosos factores, por lo que su análisis es crucial para realizar propuestas didácticas encaminadas a potenciar aquellos aspectos más relevantes. Actualmente, desde edades tempranas, aprendemos idiomas en el contexto escolar (Coyle y Férez, 2018), pero también en nuestro tiempo libre, por ejemplo, al jugar a videojuegos (Thorne, 2008), leer fan fiction (Cassany y Hernández, 2012) o formar parte de comunidades que realizan subtítulos para contenido audiovisual (Zhang y Cassany, 2016).

De hecho, ya Pendanx (1998) estableció una dicotomía entre aprendizaje en una situación formal e informal mientras que otros autores como García Santa Cecilia (1995) o Puren (1998) partían del contexto, distinguiendo entre el endolingüe o exolingüe. Atendiendo a las situaciones de aprendizaje informal, el nacimiento de Internet ha sido clave pues, como señalan Shafirova y Cassany (2017: 49) 'la tecnología digital distribuye el conocimiento de modo más inmediato, barato y ubicuo, lo cual también acelera la globalización y los contextos para usar las lenguas extranjeras'.

Sin embargo, a pesar de que se ha demostrado que no es suficiente con la asistencia a clase de lengua extranjera para adquirir un idioma, algunos autores, como Krashen (1987), han reconocido su importancia como punto de partida para que los estudiantes lleguen a usar la lengua en el mundo exterior.

En consonancia con estos planteamientos, nuestra investigación persigue un doble objetivo. Por un lado, conocer el uso de la lengua extranjera (inglés o francés) del futuro profesorado de enseñanza secundaria con familiares y amigos. Por otro lado, indagar sobre la relación que existe entre el léxico disponible y el uso de la lengua en un contexto familiar, complementando así resultados previos sobre la importancia de las estancias en el extranjero (Santos Díaz, 2015) y de la exposición en un contexto formal o académico a la lengua sobre el léxico disponible (Santos Díaz, 2018).

En ambos casos, la exposición a la lengua guarda una relación lineal con la producción léxica, de forma que los informantes que realizaron estancias largas en el extranjero -como puede ser a través de una beca Erasmus-y los que realizaron estudios relacionados con el aprendizaje de una lengua actualizaron más palabras. Por ello, se parte de la hipótesis de que las personas que usan con mayor asiduidad una lengua extranjera con familiares o amigos tendrán más facilidad no solo para proporcionar un mayor número de palabras en esa lengua, sino también en otra lengua extranjera.

\section{METOdOLOGÍA}

La recogida de datos se realizó mediante un cuestionario con preguntas asociadas al aprendizaje y uso de una lengua extranjera y una prueba de disponibilidad léxica. Esta prueba asociativa, clásica en los estudios de disponibilidad léxica, 
recoge el vocabulario sobre nueve centros de interés relacionados con temas de la vida cotidiana: 1 . Partes del cuerpo humano; 2 . La ropa; 3 . Comidas y bebidas; 4. La escuela: muebles y materiales; 5. La ciudad; 6. Medios de transporte; 7. Juegos y distracciones; 8. Profesiones y oficios y 9. Ordenadores e internet.

La metodología se aplicó siguiendo los principios establecidos por el Proyecto Panhispánico de Disponibilidad Léxica. El informante tiene que escribir las palabras que se le vengan a la mente sobre cada tema durante dos minutos (López González, 2014). La muestra estaba constituida por 171 estudiantes de postgrado de la Universidad de Málaga que eligieron realizar la prueba en inglés $(n=150)$ o francés $(n=21)$ según el idioma acreditado para acceder a los estudios de máster. Los criterios de lematización siguen las recomendaciones de Samper Padilla (1998) con ciertas salvedades debido a la adaptación a la lengua extranjera (inglés o francés).

\section{ANTECEDENTES TEÓRICOS}

El léxico es uno de los elementos cruciales para establecer comunicación en una lengua, ya sea materna o extranjera. Por esta razón, los estudios de disponibilidad léxica nacieron con fines didácticos en la década de los cincuenta del siglo pasado (Gougenheim, Michéa, Rivenc y Sauvageot, 1956) y, desde entonces, son varios los investigadores que se han preocupado por establecer puentes de conexión entre la lingüística aplicada y la enseñanza del léxico (Rodríguez y Muñoz, 2009; Palapanidi, 2012; Trigo, Romero y Santos Díaz, 2018).

Centrados en la enseñanza de segundas lenguas, la mayoría de los trabajos realizados sigue la línea trazada por Carcedo (2000), dedicada a estudiar el aprendizaje del español como segunda lengua. Por otra parte, se ha indagado sobre las bases cognitivas implícitas en los inventarios léxicos generados en L1 y L2 (Hernández y Tomé, 2017) y sobre la disponibilidad léxica en inglés (Germany y Cortés, 2000; Jiménez y Ojeda, 2009) y en francés, ambas como segunda lengua (De la Maya, 2015). Sin embargo, hasta la publicación de los trabajos de Santos Díaz $(2015 ; 2018)$ no se había prestado atención a las dos lenguas a la vez. Por ello, con nuestro estudio pretendemos seguir indagando en esta dimensión poco explorada aún.

\section{RESULTADOS Y DISCUSIÓN}

En primer lugar, se presentan los análisis descriptivos de las dos variables extralingüísticas analizadas relacionadas con el uso de la lengua con en un contexto informal: con familiares y amigos. A continuación, se muestran los resultados de la relación lineal entre el uso de la lengua y el léxico disponible.

Dado que el uso de la lengua en un contexto familiar viene marcado por la lengua materna que se habla en casa, es necesario describir la muestra objeto de estudio. La Tabla 1 muestra que el español es la lengua materna de la mayoría de los informantes $(93,6 \%)$ y de sus progenitores $(94,7 \%$ en el caso de los padres 
y $93 \%$ en el de las madres). Tan solo hay 2 informantes bilingües, de los cuales 1 tiene como lengua materna uno de los idiomas de la prueba, el inglés.

Tabla 1 Descripción de la muestra de estudio

\begin{tabular}{|l|c|c|c|c|c|c|}
\hline & \multicolumn{2}{|c|}{ Informante } & \multicolumn{2}{c|}{ Padre } & \multicolumn{2}{c|}{ Madre } \\
\hline \multicolumn{1}{|c|}{ Lengua materna } & $\mathrm{N}$ & $\%$ & $\mathrm{~N}$ & $\%$ & $\mathrm{~N}$ & $\%$ \\
\hline Español & 160 & 93,6 & 162 & 94,7 & 159 & 93,0 \\
\hline Lenguas de la prueba & & & & & 1 & 0,6 \\
\hline Otra lengua & 7 & 4,1 & 7 & 4,1 & 10 & 5,8 \\
\hline $\begin{array}{l}\text { Bilingüe (una es la lengua de la } \\
\text { prueba) }\end{array}$ & 2 & 1,2 & & & & \\
\hline Bilingüe (otras lenguas) & 2 & 1,2 & 2 & 1,2 & 1 & 0,6 \\
\hline
\end{tabular}

\subsection{USO DE LA LENGUA CON FAMILIARES Y AMIGOS}

Para poder medir la utilización de alguna lengua diferente al español, los informantes indicaron la frecuencia con la que usaban las lenguas de la prueba (inglés o francés) u otra lengua (distinta al español) tanto con familiares (se incluye pareja) como con amigos. Para ello, pudieron elegir entre 5 opciones, codificadas de 0 a 4 según una escala de Likert (nunca; alguna vez; un día a la semana; varias veces por semana y todos los días).

La Figura 1 muestra la frecuencia con la que los informantes utilizan las lenguas extranjeras de la prueba u otra lengua extranjera. En el ambiente familiar, hay 144 informantes que no usan nunca las lenguas de la prueba (inglés o francés) y 161 que no usan otra lengua extranjera diferente. Sin embargo, el uso de la lengua extranjera con los amigos es mayor ya que tan solo 97 informantes afirman no utilizar nunca las lenguas de la prueba y 143 otra lengua.

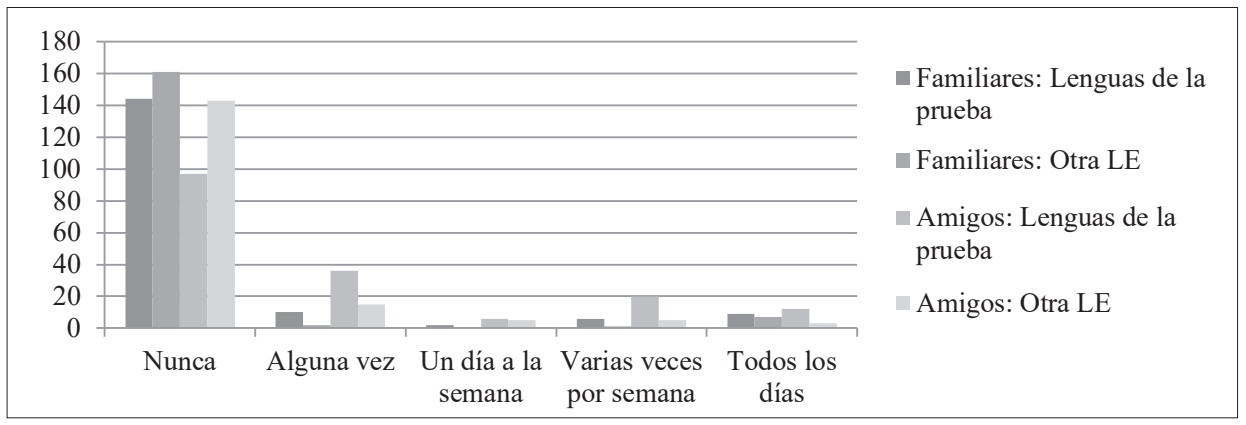

Figura 1 Diagrama de barras sobre el uso de la lengua

Si bien los resultados del uso de las lenguas extranjeras son similares, se observa una mayor utilización de las lenguas de la prueba con amigos, ya que hay 
12 informantes que la usan todos los días frente a los 9 que la usan en contexto familiar. En cambio, los datos referidos al uso diario de otra lengua extranjera se invierten: hay tan solo 3 informantes que la usan diariamente con amigos mientras que 7 afirman hacerlo con sus familiares, sobre todo porque es la lengua materna de sus progenitores.

\subsection{RELACIÓN ENTRE EL USO DE LA LENGUA Y EL LÉXICO DISPONIBLE}

La variable lingüística obtenida a través de la prueba de disponibilidad léxica mide el número de palabras que los informantes han aportado en los nueve centros de interés. Nos interesa saber en qué medida se relaciona la mayor productividad léxica con el uso de la lengua.

La Figura 2 muestra la distribución de medias de palabras según el uso de la lengua. En la parte superior, se sitúan las líneas referidas a un mayor uso de las lenguas de la prueba con respecto a otras lenguas, con lo que el léxico actualizado por esos informantes es más abundante. En el uso de otra lengua con familiares no hay ningún informante que haya marcado la opción de 'un día a la semana' con lo que la línea roja queda dividida en dos partes (para más información sobre las medias exactas en cada franja, vid. Tabla 2).

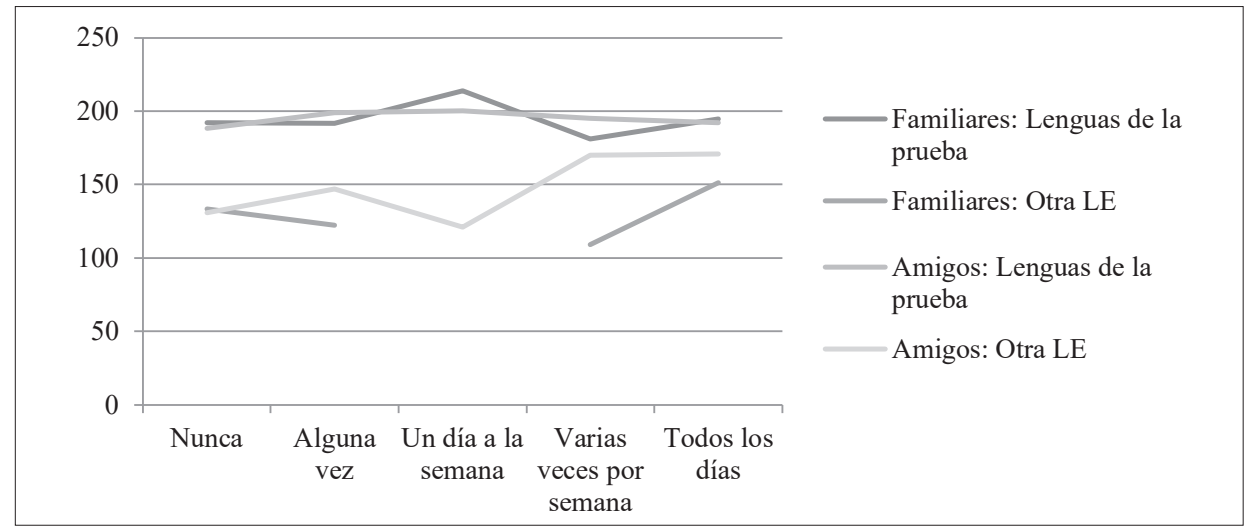

Figura 2 Media de palabras según el uso de la lengua

Para conocer la relación lineal que se produce entre el léxico y las variables extralingüísticas hemos realizado un análisis de correlación bilateral (vid. Tabla 3). El léxico guarda una relación significativa a un nivel de 0,01 con el uso de la lengua con familiares y con amigos. Mientras que en el caso del uso de otra lengua extranjera parece no tener incidencia cuando se hace en el contexto de la familia, sí lo es cuando se produce con amigos (coeficiente de Pearson de $0,206)$. La correlación más significativa se produce en el propio uso de la lengua extranjera (coeficiente de correlación de 0,449), de forma que los informantes que usan la lengua con familiares son también quienes la usan con amigos ya que el 
entorno más cercano se extiende a familiares y amigos. Esa situación se reproduce no solo con las lenguas de la prueba, sino también con otra lengua extranjera.

Tabla 2 Comparación de medias según el uso de la lengua extranjera y el léxico disponible

\begin{tabular}{|l|l|c|c|c|c|c|c|}
\hline \multirow{4}{*}{} & \multicolumn{1}{|c|}{ Frecuencia } & \multicolumn{3}{|c|}{ Familiares } & \multicolumn{3}{c|}{ Amigos } \\
\cline { 2 - 9 } & Media & N & $\begin{array}{c}\text { Desv. } \\
\text { estándar }\end{array}$ & Media & N & $\begin{array}{c}\text { Desv. } \\
\text { estándar }\end{array}$ \\
\hline \multirow{2}{*}{$\begin{array}{l}\text { Lenguas } \\
\text { de la } \\
\text { prueba }\end{array}$} & Nunca & 191,92 & 144 & 36,557 & 188,15 & 97 & 35,846 \\
\cline { 2 - 9 } & Alguna vez & 191,50 & 10 & 27,517 & 199,06 & 36 & 38,542 \\
\cline { 2 - 9 } & Un día a la semana & 214,00 & 2 & 59,397 & 200,17 & 6 & 26,317 \\
\cline { 2 - 9 } & Varias veces por semana & 181,17 & 6 & 22,912 & 195,00 & 20 & 39,029 \\
\cline { 2 - 9 } & Todos los días & 194,89 & 9 & 48,658 & 191,92 & 12 & 34,647 \\
\cline { 2 - 9 } & Total & 191,94 & 171 & 36,361 & 191,94 & 171 & 36,361 \\
\hline \multirow{5}{*}{ Otra LE } & Nunca & 133,27 & 161 & 37,517 & 130,73 & 143 & 36,086 \\
\cline { 2 - 9 } & Alguna vez & 122,50 & 2 & 53,033 & 147,13 & 15 & 35,323 \\
\cline { 2 - 8 } & Un día a la semana & & & & 121,20 & 5 & 34,528 \\
\cline { 2 - 9 } & Varias veces por semana & 109,00 & 1 & & 169,80 & 5 & 52,822 \\
\cline { 2 - 8 } & Todos los días & 151,43 & 7 & 29,826 & 171,00 & 3 & 36,756 \\
\cline { 2 - 8 } & Total & 133,74 & 171 & 37,295 & 133,74 & 171 & 37,295 \\
\hline
\end{tabular}

Tabla 3 Correlaciones bivariadas entre el léxico disponible y las variables relacionadas con el uso de la lengua

\begin{tabular}{|c|c|c|c|c|c|c|c|}
\hline & \multirow[b]{2}{*}{ Léxico } & \multicolumn{2}{|c|}{ Familiares } & \multicolumn{2}{|c|}{ Amigos } \\
\hline & & & & $\begin{array}{c}\text { Lenguas } \\
\text { de la } \\
\text { prueba }\end{array}$ & $\begin{array}{l}\text { Otra } \\
\text { LE }\end{array}$ & $\begin{array}{c}\text { Lenguas } \\
\text { de la } \\
\text { prueba }\end{array}$ & $\begin{array}{c}\text { Otra } \\
\text { LE }\end{array}$ \\
\hline \multirow{3}{*}{\multicolumn{2}{|c|}{$\begin{array}{l}\text { Léxico } \\
\text { Sig. (bilateral) } \\
\text { N }\end{array}$}} & Correlación de Pearson & 1 &, $218^{* *}$ & 0,076 &, $323^{* *}$ & $206^{* *}$ \\
\hline & & & 0,004 & 0,324 & 0 & 0,007 & \\
\hline & & 171 & 171 & 171 & 171 & 171 & \\
\hline \multirow[t]{6}{*}{ Familiares } & \multirow{3}{*}{$\begin{array}{l}\text { Lenguas } \\
\text { prueba }\end{array}$} & Correlación de Pearson & $218^{* *}$ & 1 & 0,141 & $236^{* *}$ & $449^{* *}$ \\
\hline & & Sig. (bilateral) & 0,004 & & 0,067 & 0,002 & 0 \\
\hline & & $\mathrm{N}$ & 171 & 171 & 171 & 171 & 171 \\
\hline & \multirow{3}{*}{ Otra LE } & Correlación de Pearson & 0,076 & 0,141 & 1 & $384^{* *}$ & $271^{* *}$ \\
\hline & & Sig. (bilateral) & 0,324 & 0,067 & & 0 & 0 \\
\hline & & $\mathrm{N}$ & 171 & 171 & 171 & 171 & 171 \\
\hline \multirow[t]{6}{*}{ Amigos } & \multirow{3}{*}{$\begin{array}{l}\text { Lenguas } \\
\text { prueba }\end{array}$} & Correlación de Pearson &, $323^{* *}$ & $449^{* *}$ & $271^{* *}$ &, $330^{* *}$ & 1 \\
\hline & & Sig. (bilateral) & 0 & 0 & 0 & 0 & \\
\hline & & $\mathrm{N}$ & 171 & 171 & 171 & 171 & 171 \\
\hline & \multirow{3}{*}{ Otra LE } & Correlación de Pearson & $206^{* *}$ & $236^{* *}$ &, $384^{* *}$ & 1 & $330^{* *}$ \\
\hline & & Sig. (bilateral) & 0,007 & 0,002 & 0 & & 0 \\
\hline & & $\mathrm{N}$ & 171 & 171 & 171 & 171 & 171 \\
\hline
\end{tabular}




\section{CONCLUSIONES}

La relación entre el nivel de formación en una lengua extranjera y el léxico disponible es directamente proporcional, como se ha demostrado en estudios precedentes (Germany y Cortés, 2000; Jiménez y Ojeda, 2009; Palapanidi, 2012; De la Maya, 2015; Santos Díaz, 2018).

Sin embargo, no solo la educación formal garantiza la mejora de la competencia lingüística, en general, y la léxica, en particular, de una lengua extranjera, sino que existe una amplia gama de posibilidades para su desarrollo, como puede ser la vía cibernética (Cassany y Hernández, 2012) o las estancias en el extranjero (Santos Díaz, 2018).

Estos dos estudios concuerdan con nuestros hallazgos, pues ambas vías están conectadas con el establecimiento de relaciones socioafectivas. Disponer de esta información nos ayudará a diseñar propuestas didácticas ajustadas a los intereses reales de nuestros estudiantes.

\section{REFERENCIAS BIBLIOGRÁFICAS}

Carcedo González, A. (2000) Disponibilidad léxica en español como lengua extranjera: el caso finlandés (estudio del nivel preuniversitario y cotejo con tres fases de adquisición). Humaniora, Ser. B, Tom. 238. Turku: Annales Universitatis Turkuensis. Disponible en https://dialnet. unirioja.es/servlet/articulo? codigo $=6275938$ [Consultado el 14 de mayo de 2019].

Cassany, D. y Hernández D. (2012) ¿Internet: 1; Escuela: 0? CPU-e. Revista de Investigación Educativa, 14: 126-141. Disponible en https://goo.gl/8PWY1m [Consultado el 8 de junio de 2019].

Coyle, Y. y Férez, P. A. (2018) Learning a Second Language in Pre-School: Using Dramatized Stories as a Teaching Resource. Didáctica: lengua y literatura, 30: 73-85.

De la Maya, G. (2015) El desarrollo de la competencia léxica en la adquisición del francés como segunda lengua extranjera en la ESO. Tesis doctoral: Universidad de Extremadura.

García Santa Cecilia, A. (1995) El currículo del español como lengua extranjera. Madrid: Edelsa.

Germany, P. y Cortés, N. (2000) Léxico disponible en inglés como segunda lengua en instrucción personalizada. Estudios Pedagógicos, 26: 39-50.

Gougenheim, G., Michéa, R., Rivenc, P. y Sauvageot, A. (1956) L'élaboration du francais élémentaire. París: Didier.

Hernández, N. y Tomé, C. (2017) Léxico disponible en primera y segunda lengua: bases cognitivas. En F. del Barrio (ed.) Palabras Vocabulario Léxico. La lexicología aplicada a la didáctica y a la diacronía (pp. 99-122). Venecia: Edizioni Ca’ Foscari.

Jiménez, R. M y Ojeda, J. (2009) Disponibilidad léxica en inglés como lengua extranjera en dos tipos de instrucción. Lenguaje y Textos, 30: 167-176.

Krashen, S. D. (1987) Principles and Practice in Second Language Acquisition. Hertfordshire: Prentice Hall International Ltd.

López González, A. M. (2014) Disponibilidad léxica. Teoría, método y análisis. Lodz: Universidad de Lodz.

Palapanidi, K. (2012) La aplicación de la disponibilidad léxica a la didáctica del léxico de LE. Revista Nebrija de Lingüística Aplicada, 11. Disponible en https://goo.gl/sJ4Wv7 [Consultado el 8 de junio de 2019].

Pendanx, M. (1998) Les activités d'apprentissage en classe de langue. París: Hachette F.L.E.

Puren, C. (1998) Concepts et conceptualisation en Didactique des Langues: pour une épistémologie disciplinaire. Didáctica: Lengua y Literatura, 10: 271-288. 
Rodríguez, F. J. y Muñoz, I. O. (2009) De la disponibilidad a la didáctica léxica. Tejuelo, 4: 8-18.

Santos Díaz, I. C. (2015) El impacto de las estancias en el extranjero en el léxico disponible en inglés y francés de estudiantes de postgrado. E-AESLA, 1. Disponible en http://cvc. cervantes.es/lengua/eaesla/pdf/01/32.pdf [Consultado el 8 de junio de 2019].

Santos Díaz, I. C. (2018) Incidencia del uso de la lengua extranjera (inglés y francés) en clase sobre el léxico disponible. E-AESLA, 4: 95-100. Disponible en https://cvc.cervantes.es/ lengua/eaesla/pdf/04/09.pdf [Consultado el 8 de junio de 2019].

Samper Padilla, J. A. (1998) Criterios de edición del léxico disponible: sugerencias. Lingüística, 10: 311-333.

Shafirova, L. y Cassany, D. (2017) Aprendiendo idiomas en línea en el tiempo libre. Revista de Estudios Socioeducativos. RESED, 5: 49-62.

Trigo, E., Romero, M. F. y Santos Díaz, I. C. (2018) Elaboración de un corpus cacográfico desde la disponibilidad léxica en estudiantes sevillanos. Un análisis para la enseñanza de la lengua. Revista de Lingüística y Lenguas Aplicadas, 13: 119-131.

Thorne, S. L. (2008) Mediating Technologies and Second Language Learning. En J. Corio, M. Knobel, C. Landkshear y D. Leu (eds.) Handbook of Research on New Literacies (pp. 417-448). New York: Lawrence Erlbaum.

Zhang, L. T. y Cassany, D. (2016) Fansubbing del español al chino: organización, roles y normes en la escritura colaborativa. Bid, 37, diciembre. Disponible en https://goo.gl/zCTmtd [Consultado el 8 de junio de 2019].

\title{
RELATIONSHIP BETWEEN INFORMAL FOREIGN LANGUAGE SETTING (ENGLISH AND FRENCH) AND LEXICAL COMPETENCE
}

\begin{abstract}
Learning a foreign language is sometimes limited to an academic context. However, exposure to a language in an informal context, understood as that which is carried out in a certain social environment or in a foreign country (Pendanx, 1998), plays a crucial role both in the ability to learn or acquire language and in the intrinsic and extrinsic motivation of the speakers. The purpose of this paper is to show the relationship between the vocabulary and the use of a foreign language (English or French) in a familiar context. The informants, 171 postgraduate students of the University of Malaga, answered a questionnaire that included the number of weekly hours that they used the foreign language to communicate with family and friends. In addition, to evaluate the vocabulary, they performed a lexical availability test following the recommendations of the Pan-Hispanic Project of Lexical Availability in which they evoked words related to a topic or centre of interest for two minutes. The results show that there is a significant positive correlation between the vocabulary and the variables related to the informal exposure to the foreign language. The Pearson correlation coefficient between the lexicon and the use of the language with friends is 0.323 and between the lexicon and the use of the language with relatives it is 0.218 , in both cases at a level of significance of 0.01 . Thus, the importance of informal learning in the learning of a foreign language is corroborated (Krashen, 1987).
\end{abstract}

Key words: learning a foreign language, learning context, lexical availability, vocabulary 
Inmaculada Clotilde Santos Díaz (Dra. en Innovación Educativa, profesora asociada) trabaja en la Universidad de Málaga (España). Sus líneas de investigación incluyen la disponibilidad léxica, la formación del profesorado y la enseñanza de una lengua. Correo electrónico: santosdiaz@uma.es

Ester Trigo Ibáñez (Dra. en Lengua Española y Lingüística, profesora ayudante doctora) trabaja en la Universidad de Cádiz (España). Sus intereses académicos se centran en la didáctica de la lengua, la disponibilidad léxica y los proyectos lingüísticos de centro. Correo electrónico: ester.trigo@uca.es

Manuel Francisco Romero Oliva (Dr. en Didáctica de la Lengua y la Literatura, Director de Departamento Didáctica de la Lengua y la Literatura) trabaja en la Universidad de Cádiz (España). Sus líneas de investigación se centran en la educación lingüística y literaria desde una disciplina interdisciplinar, la disponibilidad léxica y los proyectos lingüísticos de centro. Correo electrónico: manuelfrancisco.romero@uca.es 